\title{
BMJ Open Gestational weight gain and adverse pregnancy outcomes: a prospective cohort study
}

\author{
Yuelin Wu, ${ }^{1}$ Sheng Wan, ${ }^{1}$ Shengyi Gu, ${ }^{2}$ Zhengqian Mou, ${ }^{2}$ Lingling Dong, ${ }^{1}$ \\ Zhongcheng Luo (D) , Jun Zhang, ${ }^{2}$ Xiaolin Hua (D) ${ }^{1}$
}

To cite: Wu Y, Wan S, Gu S, et al. Gestational weight gain and adverse pregnancy outcomes: a prospective cohort study. BMJ Open 2020;10:e038187. doi:10.1136/ bmjopen-2020-038187

- Prepublication history and additional material for this paper are available online. To view these files, please visit the journal online (http://dx.doi. org/10.1136/bmjopen-2020038187).

YW and SW contributed equally.

Received 02 March 2020

Revised 21 July 2020

Accepted 22 July 2020

Check for updates

(C) Author(s) (or their employer(s)) 2020. Re-use permitted under CC BY-NC. No commercial re-use. See rights and permissions. Published by BMJ.

${ }^{1}$ Department of Obstetrics, Shanghai First Maternity and Infant Hospital, Shanghai, China ${ }^{2}$ Department of Obstetrics and Gynaecology, Xinhua Hospital, Shanghai Jiao Tong University School of Medicine, Shanghai, China

${ }^{3}$ Obstetrics and Gynaecology, Mount Sinai Hospital, LunenfeldTanenbaum Research Institute, Toronto, Ontario, Canada

Correspondence to Dr Xiaolin Hua; xiaolin_hua@tongji.edu.cn and Dr Jun Zhang;

zhangjun@xinhuamed.com.cn

\section{ABSTRACT}

Objective To assess the associations of gestational weight gain (GWG) in early and late pregnancy with subsequent risks of adverse pregnancy outcomes in Chinese women.

Design Prospective cohort study.

Setting Shanghai, China.

Participants We studied 2630 nulliparous singleton pregnant women with complete data on weight gain in early ( $\leq 17$ weeks of gestation) and late ( $>17$ weeks) pregnancy in the Shanghai Birth Cohort.

Methods GWG was standardised into z-scores by gestational age and categorised as low (z-score $<-1)$, normal $(-1$ to +1$)$ and high $(>1)$. The adjusted relative risks (aRRs) and 95\%Cls were estimated through logbinomial regression models. Interaction effects between GWG and some other adjustment factors were tested, further stratified analyses were performed separately where interaction terms were significant.

Outcome measures Adverse maternal and neonatal outcomes.

Results Independent from GWG in late pregnancy, higher GWG in early pregnancy was associated with higher risks of gestational diabetes mellitus (aRR: 1.66 ; $95 \% \mathrm{Cl}: 1.11$ to 2.48), caesarean section (aRR: $1.21 ; 95 \% \mathrm{Cl}: 1.05$ to 1.39 ) and prolonged hospitalisation (aRR: 1.56 ; $95 \% \mathrm{Cl}: 1.03$ to 2.38). Higher GWG in late pregnancy was independently associated with higher risks of caesarean section (aRR: 1.24; $95 \% \mathrm{Cl}: 1.09$ to 1.41), large for gestational age (aRR: 2.01 ; $95 \% \mathrm{Cl}: 1.50$ to 2.7 ) and macrosomia (aRR: $1.90 ; 95 \% \mathrm{Cl}$ : 1.30 to 2.78). In addition, the risk of gestational hypertension increased significantly with increased total GWG (aRR: 1.78; $95 \% \mathrm{Cl}: 1.14$ to 2.76 ). The effects of GWG in late pregnancy on maternal and neonatal outcomes were significantly different between the women bearing a female and the women bearing male fetus.

Conclusion The GWG associations with adverse pregnancy outcomes differ at early and late pregnancy, and there may be effect modification by fetal sex in the association of GWG in late pregnancy with some pregnancy outcomes.

\section{INTRODUCTION}

Gestational weight gain (GWG) has been associated with pregnancy outcomes. Insufficient weight gain has been linked with increased risks of low birth weight, small for gestational

\section{Strengths and limitations of this study}

- Weight gain data collected before and during pregnancy enabled us to investigate the effect of timing of weight gain on the outcomes.

- The use of z-scores instead of original weight gain value to account for the gestational-age-dependent nature of gestational weight gain allowed us to differentiate the effect caused by weight gain from the effect caused by duration of pregnancy.

- Effect modification by fetal sex was investigated.

- Only short-term rather than long-term pregnancy outcomes were investigated.

- Prepregnancy weight was self-reported rather than measured.

age (SGA) and preterm birth, while excessive weight gain has been associated with large for gestational age (LGA), gestational diabetes mellitus (GDM), pre-eclampsia, preterm birth, caesarean section, infant mortality and childhood obesity. ${ }^{12}$ However, although women are routinely weighed in clinical settings and receive GWG advice, ${ }^{34}$ a high proportion of pregnant women gain above or below GWG weight ranges recommended by the guidelines. ${ }^{5}$ Based on data collected from 23 studies involving more than 1.3 million women, GWG was below or above the weight gain range suggested by Institute of Medicine (IOM) guidelines in 23\% and $47 \%$ of pregnancies, respectively, ${ }^{1}$ and the prevalence of excess GWG appears to be on the rise. $^{6}$

It is well established that total GWG affects pregnancy outcomes. ${ }^{7}$ Some studies suggest that GWG during early pregnancy may be more important than GWG at late pregnancy for developing certain pregnancy outcomes such as GDM and adverse cardiometabolic profile in the offspring. ${ }^{8-11}$ Overall, studies examining associations of early GWG with perinatal outcomes have been relatively few, and these studies have often not accounted 
for the effects of weight gain during other periods of pregnancy. ${ }^{12}$

In a Chinese population-based study, our objective was to explore the association of GWG during early and late pregnancy with maternal and neonatal outcomes .

\section{MATERIALS AND METHODS}

\section{Study design and data source}

This prospective cohort study is based on the recently developed Shanghai Birth Cohort (SBC), which has been described in details elsewhere. ${ }^{13}$ Briefly, the SBC is a prospective observational study conducted in Shanghai, China, aiming to examine the factors affecting fecundability, pregnancy outcomes, child growth and development, and risks of diseases. The cohort recruited 4127 women in preconception care (701) or early antenatal care (3426). Written informed consent was obtained from the participants. The data were collected between $1^{\text {st }}$ September 2013 and $31^{\text {st }}$ November 2016, resulting in 3699 live births. The collected data included maternal demographical characteristics, health behaviours, reproductive history, as well as clinical information related to pregnancy, birth and pregnancy outcomes.

\section{Study population}

The present study collected the data from all singleton pregnancies in women with age $\geq 20$ years old who started antenatal care before 17 weeks of gestation and delivered at $\geq 28$ weeks of gestation with data available on weight gains in early and late gestation in the SBC.

Gestational age was estimated based on the date of last menstruation period and confirmed by first trimester ultrasound date. The eligible data collected in this study were obtained from: (1) self-reported prepregnancy weight $(\mathrm{kg}),(2)$ weight and height $(\mathrm{cm})$ measured in early pregnancy (17 weeks of gestation or less) and (3) weight measured within the last week of pregnancy. Subjects were excluded if: (1) weight in early pregnancy $<30 \mathrm{~kg}$ or $>350 \mathrm{~kg}$ or (2) z-score of GWG $<-4.0$ or $>4.0$, the methods used in the study were similar to the study conducted by Johansson et al. ${ }^{14}$ Women with pre-existing medical conditions such as pregestational diabetes, hypothyroidism or hyperthyroidism (affecting GWG) ${ }^{15}$ and heart/liver/kidney diseases were also excluded.

\section{Weight measurements}

Prepregnancy weight $(\mathrm{kg})$ was based on self-reporting, while weight at early pregnancy and at delivery was routinely measured to the nearest $0.1 \mathrm{~kg}$ using the available electronic weighing device in the prenatal care clinics. Height $(\mathrm{cm})$ at the first prenatal visit was routinely measured to the nearest $0.1 \mathrm{~cm}$ using the available electronic stadiometer in the hospital. Prepregnancy body mass index (BMI; $\mathrm{kg} / \mathrm{m}^{2}$ ) was calculated as prepregnancy weight $(\mathrm{kg})$ divided by height $(\mathrm{m})^{2}$ and categorised as underweight $\left(<18.5 \mathrm{~kg} / \mathrm{m}^{2}\right)$, normal weight (18.5 to
$24.9 \mathrm{~kg} / \mathrm{m}^{2}$ ), overweight (25.0 to $29.9 \mathrm{~kg} / \mathrm{m}^{2}$ ) and obese $\left(\geq 30.0 \mathrm{~kg} / \mathrm{m}^{2}\right) .^{16}$

The 2009 IOM recommendations suggested $0.5-2 \mathrm{~kg}$ weight gain for women in the first trimester $(0-13 \text { weeks })^{5}$ and the $50^{\text {th }}$ centile GWG for women at gestational age $\leq 17$ weeks is below $2 \mathrm{~kg}$ according to the INTERGROWTH-21 ${ }^{\text {st }}$ Project. ${ }^{2}$ However, early pregnancy in this study was defined as gestational age $\leq 17$ weeks so as to include virtually all women who started the first antenatal care in the hospital. ${ }^{17}$ In addition, the 2009 IOM recommendations suggested total GWG of 12.5-18 kg for women with prepregnancy BMI less than 18.5 (underweight); $11.5-16 \mathrm{~kg}$ for those with BMI of 18.5-24.9 (normal weight); 7-11.5 kg for those with an initial BMI of 25.0-29.9 (overweight) and 5-9 kg for those with an initial BMI greater than 30.0 (obese) ${ }^{5}$ In this study, due to the sporadic number of obese women, we analyse them together with overweight women. We examined GWG in early pregnancy (the last weight measured $\leq 17$ weeks minus prepregnancy weight) and late pregnancy (last measurement of weight prior to delivery minus the last weight measured $\leq 17$ weeks). Total GWG was calculated as last measurement of weight before delivery minus prepregnancy weight. All GWG values were standardised into z-scores by gestational age, stratified by BMI categories. The means and SD of GWGs in early pregnancy and late pregnancy were used to convert the GWG values into z-scores. All GWG z-scores were first examined as continuous variables, and then categorised as $<-1.0$ (below), -1.0 to +1.0 (average) and $>+1.0$ (above) in data analyses. Previous studies suggested different associations of gestational stagespecific weight gain with maternal and neonatal outcomes. ${ }^{8}$ For example, weight gain in early pregnancy is associated with offspring BMI, whereas weight gain in mid pregnancy tended to be associated with the offspring's metabolic and inflammatory biomarkers. ${ }^{58}$ Thus, to disentangle the associations of other periods of GWG with pregnancy outcomes from GWG in specific periods, we restricted the analyses for GWG in early pregnancy in women whose GWGs in late pregnancy were average $(-1.0$ to +1.0$)$. Similarly, the analyses for late pregnancy weight gain were restricted to women with weight gain value in early pregnancy within -1.0 to +1.0 .

\section{Covariates}

Covariables included fetal sex, maternal age (20-34, $\geq 35$ years), parity $(0, \geq 1)$, prepregnancy BMI categories (underweight, normal and overweight/obese), alcohol/ tobacco use (yes or no), GDM (yes or no), gestational hypertension (yes or no) and length of gestation (28-36, $\geq 37$ weeks).

\section{Outcomes}

The outcomes included GDM, pregnancy-induced hypertension (PIH), caesarean section, preterm birth, neonatal intensive care unit admission, neonatal prolonged hospitalisation ( $\geq 5$ days), severe neonatal outcomes, neonatal hyperbilirubinemia ( $\leq 12 \mathrm{mg} / \mathrm{dL})$, LGA, SGA, macrosomia ( $>4000 \mathrm{~g})$ and low birth weight $(<2500 \mathrm{~g})$. 
All women received a $75 \mathrm{~g}$ Oral Glucose Tolerance Test during 24-28 weeks of gestation. GDM was diagnosed according to the International Association of Diabetes and Pregnancy Study Groups (IADPSG) criteria: if anyone had the glucose values fell at or above the following thresholds: fasting $5.1 \mathrm{mmol} / \mathrm{L}, 1$ hour $10.0 \mathrm{mmol} / \mathrm{L}$ and 2 hour $8.5 \mathrm{mmol} / \mathrm{L}$. PIH was defined as de novo hypertension (systolic blood pressure $\geq 140 \mathrm{~mm} \mathrm{Hg}$ or diastolic blood pressure $\geq 90 \mathrm{~mm} \mathrm{Hg}$ ) after 20 weeks of gestation. Preterm birth was defined as gestational age at delivery $<37$ weeks. SGA was defined as birth weight $\leq 10^{\text {th }}$ percentile, and LGA as birth weight $\geq 90^{\text {th }}$ percentile according to Chinese sex-specific and gestational age-specific birth weight standards. ${ }^{18}$ Severe neonatal outcomes included death, 5 min APGAR Score $<7$, hypoglycaemic $(<40 \mathrm{mg} / \mathrm{dL})$, sepsis, cardiopulmonary resuscitation or ventilator support within 24 hours after birth, severe respiratory disorders (respiratory distress syndrome or transient tachypnea of the newborn), serious birth defects, seizures, necrotising enterocolitis and hypoxicischaemic encephalopathy.

\section{Statistical analyses}

Maternal demographic characteristics and clinical factors were compared across GWG groups. Continuous variables were described by mean with SD or median with IQR. Categorical variables were described by frequencies (\%). Analysis of variance or Kruskal-Wallis $\mathrm{H}$ tests were performed for continuous data, and $\chi^{2}$ tests or Fisher's exact tests were performed for categorical data.

The incidence of adverse pregnancy outcomes were examined among three GWG groups. Multivariate logbinomial regression models were used to estimate the unadjusted relative risks (RRs), adjusted RRs (aRRs) and 95\% CIs of adverse pregnancy outcomes across GWG groups. Regression model for maternal outcomes were adjusted for only covariables with $\mathrm{p}<0.2$ (maternal age, parity, prepregnancy BMI, alcohol/tobacco use, fetal sex and length of gestation). Women with GDM or PIH have long been known to be at increased risk for adverse neonatal outcomes, including neonatal intensive care admission, caesarean section, preterm delivery $<37$ weeks and neonatal morbidity. ${ }^{1920}$ As a result, neonatal outcomes models were further adjusted for GDM and pregnancyinduced hypertension in addition to the afore-mentioned factors. The effects between weight gain and fetal sex and other covariates on adverse neonatal outcomes were also investigated. Interaction effects between GWG and fetal sex and other covariates (parity, maternal prepregnancy BMI and maternal age) on adverse maternal and neonatal outcomes were also tested.

All analyses were performed using the Statistical Analysis System (SAS) for Windows, V.9.4 (SAS Institute). P value $<0.05$ was considered statistically significant.

\section{Patient and public involvement}

No patients were involved in the design, or conduct, or reporting, or dissemination plans of our research.

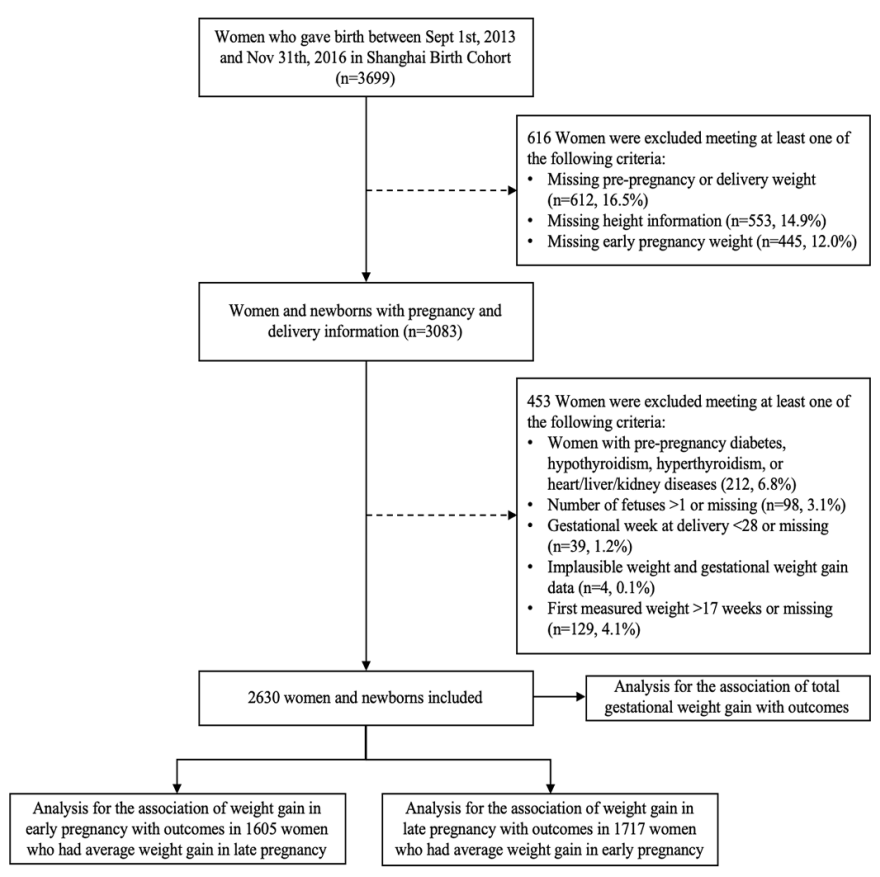

Figure 1 Study flow chart.

\section{RESULTS}

\section{Study population and characteristics}

A total of 2630 pregnant women met the study inclusion criteria (figure 1). The characteristics of all pregnant women in the study by total GWG are shown in table 1 . Among them, 1605 women who gained average weight in late pregnancy ( $z$-score -1 to +1 ) were analysed for the association of GWG in early pregnancy with the outcomes (online supplementary table S1); while 1717 women who gained average weight in early pregnancy were analysed for the association of GWG in late pregnancy with the outcomes (online supplementary table S2). It should be noticed that for the two analytic datasets, the reference group was the same group of women who had average GWG in both early and late pregnancy $(n=1233)$.

\section{Weight gain during early pregnancy}

The risks of maternal and neonatal outcomes for GWG in early pregnancy are presented in figure $2 \mathrm{~A}$ and online supplementary table S3. Lower GWG was not significantly associated with pregnancy outcomes, compared with the average GWG. In contrast, the risks of GDM $(\mathrm{aRR}=1.66$; 95\% CI: 1.11 to 2.48$)$, caesarean section $(\mathrm{aRR}=1.21$; 95\% CI: 1.05 to 1.39 ) and prolonged hospitalisation (aRR=1.56; $95 \%$ CI: 1.03 to 2.38$)$ were higher in the group with GWG above average in early pregnancy. No significant interactions between GWG in early pregnancy and covariates were observed.

\section{Weight gain during late pregnancy}

Associations of GWG in late pregnancy with perinatal outcomes are presented in figure $2 \mathrm{~B}$ and online supplementary table S4. In contrast to early pregnancy, the risks for GDM and SGA decreased significantly with increased GWG in late pregnancy, whereas the risks for LGA and 
Table 1 Characteristics of pregnant women in the study cohort $(n=2630)$

\begin{tabular}{|c|c|c|c|c|c|}
\hline \multirow[b]{2}{*}{ Characteristic } & \multirow[b]{2}{*}{ Total } & \multicolumn{4}{|c|}{ Total GWG } \\
\hline & & $\begin{array}{l}\text { Below } \\
(n=392)\end{array}$ & $\begin{array}{l}\text { Average } \\
(\mathrm{n}=1842)\end{array}$ & $\begin{array}{l}\begin{array}{l}\text { Above } \\
(n=396)\end{array}\end{array}$ & $P$ value \\
\hline Maternal age (year), mean $\pm S D$ & $29.4 \pm 3.6$ & $29.9 \pm 3.8$ & $29.4 \pm 3.5$ & $28.5 \pm 3.6$ & $<0.0001$ \\
\hline Maternal age $\geq 35$ years, $n(\%)$ & $241(9.2)$ & $51(13)$ & $169(9.2)$ & $21(5.3)$ & 0.0002 \\
\hline Nulliparous, $n(\%)$ & $2216(84.3)$ & $325(82.9)$ & $1532(83.2)$ & $359(90.7)$ & 0.0027 \\
\hline Education, university degree and above, $\mathrm{n}(\%)$ & $2404(91.5)$ & $362(92.6)$ & $1702(92.5)$ & $340(85.7)$ & 0.0007 \\
\hline ART, n (\%) & $55(2.1)$ & $9(2.3)$ & $37(2)$ & $9(2.3)$ & 0.9834 \\
\hline Tobacco smoking, n (\%) & $72(2.7)$ & $7(1.8)$ & $41(2.2)$ & $24(6.1)$ & $<0.0001$ \\
\hline Alcohol use, $n(\%)$ & $338(12.9)$ & $52(13.3)$ & $240(13)$ & $46(11.6)$ & 0.7221 \\
\hline Prepregnancy BMI $\left(\mathrm{kg} / \mathrm{m}^{2}\right)$, mean \pm SD & $21.3 \pm 3.0$ & $21.7 \pm 3.1$ & $21.2 \pm 3.0$ & $21.5 \pm 2.9$ & 0.0139 \\
\hline \multicolumn{6}{|l|}{ Prepregnancy BMI categories, $\mathrm{n}(\%)$} \\
\hline Underweight $\left(<18.5 \mathrm{~kg} / \mathrm{m}^{2}\right)$ & $396(15.1)$ & $54(13.8)$ & $292(15.9)$ & $50(12.6)$ & 0.7530 \\
\hline Normal weight $\left(18.5-24.9 \mathrm{~kg} / \mathrm{m}^{2}\right)$ & $1980(75.3)$ & $298(76)$ & $1372(74.5)$ & $310(78.3)$ & \\
\hline Overweight and obese $\left(\geq 25 \mathrm{~kg} / \mathrm{m}^{2}\right)$ & $254(9.7)$ & $40(10.2)$ & $178(9.7)$ & $36(9.1)$ & \\
\hline $\begin{array}{l}\text { Gestational age at the first prenatal visit (week), } \\
\text { median (IQR) }\end{array}$ & $15(3)$ & $14(3)$ & $15(3)$ & $15(3)$ & 0.1986 \\
\hline \multicolumn{6}{|c|}{ GWG in early pregnancy by $\mathrm{BMI}$ categories $(\mathrm{kg})$, mean $\pm \mathrm{SD}$} \\
\hline All women & $2.2 \pm 2.9$ & $-0.2 \pm 2.8$ & $2.1 \pm 2.2$ & $4.9 \pm 3.2$ & $<0.0001$ \\
\hline Underweight $\left(<18.5 \mathrm{~kg} / \mathrm{m}^{2}\right)$ & $2.5 \pm 2.4$ & $0.9 \pm 1.9$ & $2.4 \pm 2.0$ & $4.8 \pm 3.0$ & $<0.0001$ \\
\hline Normal weight $\left(18.5-24.9 \mathrm{~kg} / \mathrm{m}^{2}\right)$ & $2.3 \pm 2.8$ & $0.0 \pm 2.8$ & $2.2 \pm 2.2$ & $4.9 \pm 3.1$ & $<0.0001$ \\
\hline Overweight and obese $\left(\geq 25 \mathrm{~kg} / \mathrm{m}^{2}\right)$ & $1.4 \pm 3.6$ & $-2.2 \pm 3.3$ & $1.5 \pm 2.6$ & $4.7 \pm 4.4$ & $<0.0001$ \\
\hline \multicolumn{6}{|c|}{ GWG in late pregnancy by BMI categories $(\mathrm{kg})$, mean $\pm \mathrm{SD}$} \\
\hline All women & $13.0 \pm 3.5$ & $8.2 \pm 3.0$ & $12.6 \pm 2.9$ & $17.8 \pm 3.6$ & $<0.0001$ \\
\hline Underweight $\left(<18.5 \mathrm{~kg} / \mathrm{m}^{2}\right)$ & $13.0 \pm 3.5$ & $8.9 \pm 1.9$ & $12.8 \pm 2.5$ & $18.2 \pm 3.8$ & $<0.0001$ \\
\hline Normal weight $\left(18.5-24.9 \mathrm{~kg} / \mathrm{m}^{2}\right)$ & $12.9 \pm 4.0$ & $8.4 \pm 3.0$ & $12.8 \pm 2.8$ & $17.8 \pm 3.6$ & $<0.0001$ \\
\hline Overweight and obese $\left(\geq 25 \mathrm{~kg} / \mathrm{m}^{2}\right)$ & $11.0 \pm 4.9$ & $5.8 \pm 3.3$ & $10.8 \pm 3.8$ & $17.4 \pm 3.8$ & $<0.0001$ \\
\hline \multicolumn{6}{|c|}{ GWG in whole pregnancy by BMI categories $(\mathrm{kg})$, mean $\pm \mathrm{SD}$} \\
\hline All women & $15.0 \pm 4.9$ & $8.1 \pm 2.6$ & $14.8 \pm 2.7$ & $22.7 \pm 2.9$ & $<0.0001$ \\
\hline Underweight $\left(<18.5 \mathrm{~kg} / \mathrm{m}^{2}\right)$ & $15.4 \pm 4.2$ & $9.7 \pm 1.6$ & $15.2 \pm 2.3$ & $23.1 \pm 3.0$ & $<0.0001$ \\
\hline Normal weight $\left(18.5-24.9 \mathrm{~kg} / \mathrm{m}^{2}\right)$ & $15.2 \pm 4.7$ & $8.4 \pm 2.1$ & $15.0 \pm 2.5$ & $22.7 \pm 2.8$ & $<0.0001$ \\
\hline Overweight and obese $\left(\geq 25 \mathrm{~kg} / \mathrm{m}^{2}\right)$ & $12.3 \pm 6.1$ & $3.7 \pm 2.4$ & $12.3 \pm 3.7$ & $22.0 \pm 3.6$ & $<0.0001$ \\
\hline Gestational age at delivery (week), median (IQR) & $39(2)$ & $39(2)$ & $39(2)$ & $39(2)$ & 0.0793 \\
\hline Birth weight $(\mathrm{g})$, mean $\pm \mathrm{SD}$ & $3376 \pm 450$ & $3238 \pm 422$ & $3370 \pm 438$ & $3540 \pm 480$ & $<0.0001$ \\
\hline Male infant, $\mathrm{n}(\%)$ & $1334(51.4)$ & $194(50.4)$ & $945(52)$ & $195(49.5)$ & 0.7951 \\
\hline
\end{tabular}

ART, assisted reproductive technology; BMI, body mass index; GWG, gestational weight gain.

macrosomia increased. Weight gain above average was correlated with a higher risk for caesarean section (aRR=1.24; $95 \%$ CI: 1.09 to 1.41$)$ in late pregnancy. In addition, higher GWG showed a protective effect against neonatal hyperbilirubinemia (aRR $=0.64 ; 95 \%$ CI: 0.43 to $0.94)$.

Significant interactions were identified between GWG in late pregnancy and fetal sex. Figure 3 and online supplementary table S5 show the associations of late pregnancy weight gain with pregnancy outcomes stratified by fetal sex. The risks for LGA and caesarean section significantly increased in women with higher GWG in late pregnancy, but the risk of GDM increased with less GWG, regardless of fetal sex. However, higher risks of gestational hypertension ( $\mathrm{aRR}=2.31$; $95 \%$ CI: 1.08 to 4.95$)$ were only observed in women bearing a female fetus with higher GWG. Conversely, higher GWG in late pregnancy was associated with GDM ( $\mathrm{aRR}=0.30 ; 95 \%$ CI: 0.10 to 0.96$)$, neonatal hyperbilirubinemia (aRR $=0.46 ; 95 \%$ CI: 0.24 to 0.89 ) and macrosomia ( $\mathrm{aRR}=2.05 ; 95 \%$ CI: 1.27 to 3.31 ) for women bearing a boy, but not for women bearing a girl, indicating effect modifications by fetal sex. But when stratified by fetal sex, the lower risk of SGA with higher GWG was no longer observed. 


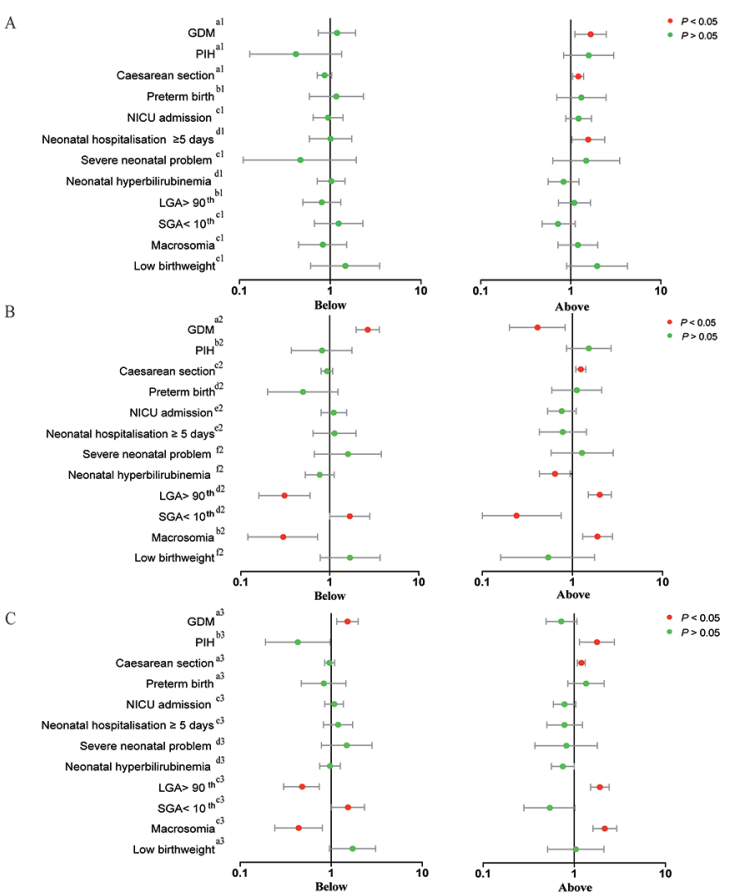

Figure 2 Associations of GWG with pregnancy outcomes. (A) Associations of early GWG ( $\leq 17$ weeks) with pregnancy outcomes. (B) Associations of late GWG (>17 weeks) with pregnancy outcomes. (C) Associations of total GWG with pregnancy outcomes. Average GWG group as the reference. ${ }^{a 1}$ The analysis was adjusted for maternal age, parity, prepregnancy BMI and length of gestation. ${ }^{\mathrm{b} 1}$ The analysis was adjusted for parity, prepregnancy BMI, fetal sex, GDM and $\mathrm{PIH}$. ${ }^{\mathrm{c}}$ The analysis was adjusted for prepregnancy BMI, fetal sex, alcohol/tobacco use, length of gestation, GDM and $\mathrm{PIH} .{ }^{\mathrm{d} 1}$ The analysis was adjusted for maternal age, alcohol/ tobacco use, GDM and PIH. ${ }^{2}$ The analysis was adjusted for maternal age and prepregnancy BMI. ${ }^{\text {b2 The analysis was }}$ adjusted for parity, prepregnancy BMI, fetal sex and length of gestation. ${ }^{\mathrm{c} 2}$ The analysis was adjusted for maternal age, parity, prepregnancy BMI, GDM and $\mathrm{PIH} .{ }^{\mathrm{d} 2}$ The analysis was adjusted for parity, prepregnancy BMI, fetal sex, GDM and $\mathrm{PIH} .{ }^{\text {e2 }}$ The analysis was adjusted for parity, prepregnancy $\mathrm{BMI}$, alcohol/tobacco use, length of gestation, GDM and $\mathrm{PIH} .{ }^{\mathrm{f}}$ The analysis was adjusted for maternal age, alcohol/ tobacco use and length of gestation. ${ }^{23}$ The analysis was adjusted for maternal age, parity, prepregnancy BMI, alcohol/ tobacco use and fetal sex. ${ }^{\text {b3 }}$ The analysis was adjusted for parity, prepregnancy BMI and length of gestation. ${ }^{\mathrm{c} 3}$ The analysis was adjusted for parity, prepregnancy BMI, length of gestation, GDM and PIH. ${ }^{\mathrm{d} 3}$ The analysis was adjusted for maternal age, parity and length of gestation. ${ }^{\text {e3 }}$ The analysis was adjusted for prepregnancy BMI, fetal sex, length of gestation, GDM and PIH. BMI, body mass index; GDM, gestational diabetes mellitus; GWG, gestational weight gain; LGA, large for gestational age; NICU, neonatal intensive care unit; PIH, pregnancy-induced hypertension; SGA, small for gestational age.

\section{Total weight gain during pregnancy}

Figure 2C and online supplementary table S6 show results for pregnancy outcomes by total GWG. The effect sizes of GWG in late pregnancy on pregnancy outcomes were almost identical with the effect sizes of total GWG. Similar
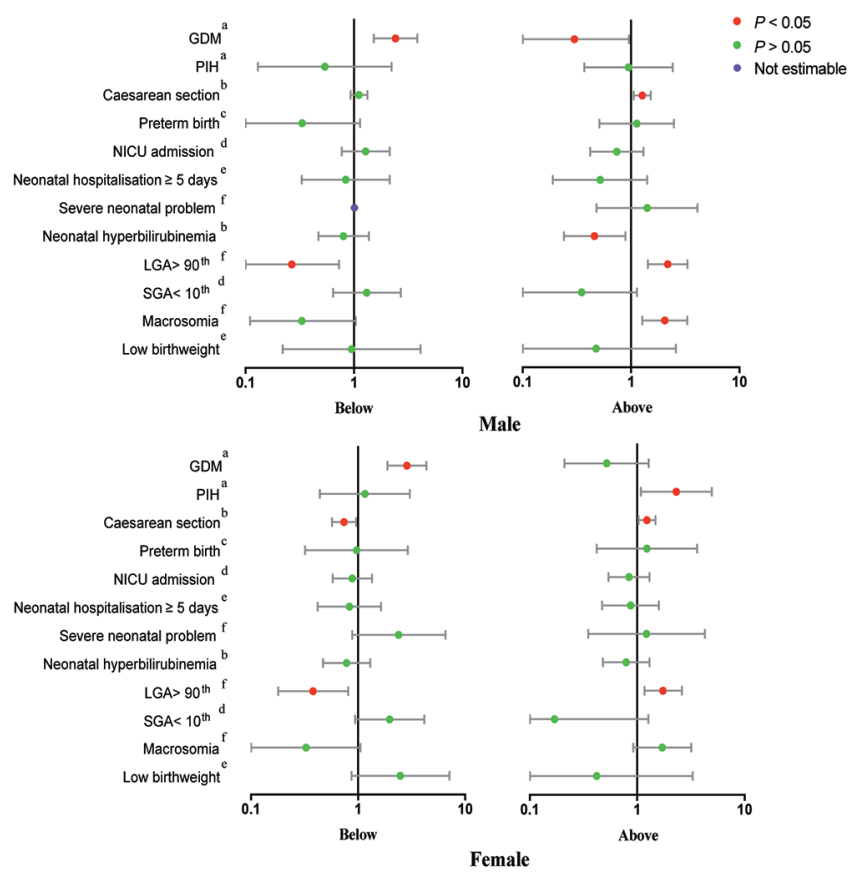

Figure 3 Associations of late GWG (>17 weeks) with pregnancy outcomes, stratified by fetal sex. Average GWG

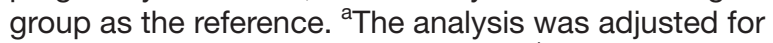
maternal age and prepregnancy BMI. ' $T$ The analysis was adjusted for maternal age, parity, prepregnancy BMI, alcohol/ tobacco use and length of gestation. ${ }^{\mathrm{C}}$ The analysis was adjusted for maternal age, prepregnancy BMI, GDM and $\mathrm{PIH} .{ }^{\mathrm{d}}$ The analysis was adjusted for parity, prepregnancy $\mathrm{BMI}$, alcohol/tobacco use and length of gestation. ${ }^{\mathrm{e}}$ The analysis was adjusted for parity, alcohol/tobacco use, length of gestation, GDM and PIH. ${ }^{f}$ The analysis was adjusted for parity, prepregnancy BMI, length of gestation, GDM and PIH. BMI, body mass index; GDM, gestational diabetes mellitus; GWG, gestational weight gain; LGA, large for gestational age; NICU, neonatal intensive care unit; $\mathrm{PIH}$, pregnancy-induced hypertension; SGA, small for gestational age.

to late pregnancy, the risks for LGA, macrosomia and gestational hypertension increased significantly with increased total GWG. Higher GWG was also linked with a higher risk for caesarean section ( $\mathrm{aRR}=1.78$; 95\% CI: 1.14 to 2.76$)$. Moreover, total GWG below the average was associated with higher risks of GDM ( $\mathrm{aRR}=1.51 ; 95 \% \mathrm{CI}: 1.15$ to 1.98$)$ and SGA (aRR=1.53; 95\% CI: 1.01 to 2.32$)$. No significant interactions between total GWG and other covariates were identified.

\section{DISCUSSION \\ Main findings}

In this study, we found different associations of gestational stage-specific weight gain with maternal and neonatal outcomes. Of those, independent of GWG in late pregnancy, higher GWG in early pregnancy was associated with higher risks of GDM, caesarean section and neonatal prolonged hospitalisation. 


\section{Strengths and limitations}

There are strengths in our study. In the study, some improvements have been made when compared with other studies reported in the literature. First, the SBC database contains detailed clinical data including prepregnancy weight, weight measurements during pregnancy and pregnancy weight measurements before delivery. This made it possible to study GWG in both early and late pregnancy. In addition, it is difficult to disentangle the effects of GWG on adverse pregnancy outcomes from the effects of the gestation duration, because GWG is highly correlated with the gestational duration. However, the use of GWG z-scores in our study can overcome this limitation. This method ensured that the weight gain of women who experience adverse pregnancy outcomes would be compared with the weight gain of women without adverse outcomes at the same point in pregnancy. ${ }^{141}$

There are also limitations in our study. We only investigated the short-term perinatal outcomes. Recently, researchers have linked an individual's susceptibility to chronic disease such as cardiometabolic disease and obesity in later life to events during the intrauterine phase of development. ${ }^{822}{ }^{23}$ Further studies on long-term outcomes would provide important evidence regarding the associations between chronic diseases and events during the intrauterine phase.

\section{Interpretation}

Associations between insufficient or excessive weight gain during the whole pregnancy and maternal and child health outcomes have been well described. ${ }^{12} 7 \mathrm{~A}$ meta-analysis of pooled 1309136 participant data from 23 cohort studies showed that women who gained high weight were more likely to have LGA, caesarean section and macrosomia, while women who gained less weight were at higher risk of SGA. ${ }^{1}$ These findings are in line with the association of maternal weight gain with adverse pregnancy outcomes in our study.

There is growing recognition that the impacts of gestational stage-specific weight gain on pregnancy outcomes may vary. ${ }^{89222425}$ GWG in early pregnancy largely reflects maternal fat deposition, whereas GWG, thereafter, is mainly attributed to maternal and amniotic fluid expansion, and growth of the fetus, placenta and uterus. ${ }^{5}$ In this study, mothers with increased fat deposition during pregnancy may affect the adiposity of the offspring by higher placental transfer of nutrients, such as glucose and free fatty acids, which may lead to maternal pregnancy complications, such as GDM, and permanent fetal and childhood adaptations in appetite, energy metabolism and neuroendocrine function. ${ }^{25}{ }^{26}$ Therefore, GWG in early pregnancy, prior to the development of pregnancy outcomes, might be as or more important than GWG in late pregnancy with respect to pregnancy outcomes. ${ }^{9}{ }^{14}$ A study of 5908 Netherlands mother-offspring pairs reported that higher weight gain in early pregnancy was associated with an adverse cardiometabolic profile in the offspring. ${ }^{8}$ Similarly, a study of 5154 UK mother-offspring pairs showed that GWG in the first 14 weeks tended to be incrementally associated with offspring BMI, waist circumference and fat mass in children at age 9 years, but after 14 weeks of gestation, only high levels of GWG were associated with offspring's adiposity measures, highlighting the importance of the timing of weight gain in pregnancy. ${ }^{24}$

Studies to clarify the relationship between gestational stage-specific weight gain and adverse pregnancy outcomes have been sporadic. A study of Korean pregnant women found that GWG velocity at early pregnancy was significantly associated with GDM, gestational hypertension, caesarean section, LGA and macrosomia. ${ }^{12}$ However, the analyses have not accounted for the effects of weight gain in other periods of pregnancy. In contrast, our analyses for early/late pregnancy GWG were restricted to women whose GWG z-scores in other pregnancy stage were normal, and thus the observed associations are independent of GWG in different periods. Our data from a large population-based Chinese Cohort in Shanghai showed that higher, but not lower, maternal GWG in early pregnancy was associated with increased risks of adverse pregnancy outcomes, including GDM, caesarean section and prolonged hospitalisation. In late pregnancy, low weight gain was associated with GDM as well as SGA, and high weight gain was associated with caesarean section, LGA and macrosomia.

High GWG in early pregnancy has been associated with an increased risk of GDM, while there are some inconsistent data concerning the associations of GWG in second and third trimester or the whole pregnancy with GDM. ${ }^{11}$ 27-29 Our study presents results that are inconsistent with results produced by other studies ${ }^{12} 2730$ indicating that higher GWG in early pregnancy may increase the risk of developing GDM, but higher GWG in late pregnancy shows a reversed association. The discrepancy might be due to that women diagnosed with GDM might have undergone weight control interventions such as prescribed diet and physical exercise after the GDM diagnosis. Avoiding high weight gain in early pregnancy may prevent GDM, and health professionals who assist prenatal care might consider pre-emptive actions in highrisk pregnant women.

The risk of gestational hypertension increases significantly with higher maternal total GWG. A study of 29861 women from 25 hospitals in USA showed that early weight gain over the 2009 IOM recommendation was shown to be associated with the development of gestational hypertension. ${ }^{31}$ In a study of 101259 women with chronic hypertension, women who gained the amount of weight above the GWG range recommended by IOM guidelines were at increased risks of eclampsia. ${ }^{32}$ Given the known vascular permeability and decreased plasma oncotic pressure that accompanies pre-eclampsia and its association with rapid weight gain, ${ }^{33}$ excessive GWG may be a cause of hypertensive disease of pregnancy.

Total GWG on average $(-1$ to +1$)$ in our study was $15.2 \pm 2.3 \mathrm{~kg}$ for women with prepregnancy BMI less than 18.5 (underweight) and $15.0 \pm 2.5 \mathrm{~kg}$ for those who with 
prepregnancy BMI of 18.5-24.9 (normal weight), which corresponded to the 2009 IOM recommendations. Specifically, the 2009 IOM recommendations suggested GWG of $12.5-18 \mathrm{~kg}$ for underweight women and $11.5-16 \mathrm{~kg}$ for normal weight women. ${ }^{5}$ However, due to the sporadic number of obese women, we analysed them together with overweight women. Total GWG on average was 12.3 \pm 3.7 $\mathrm{kg}$ for women with prepregnancy BMI greater than 25.0 (overweight and obese) in our study, which in general was higher than the 2009 IOM recommended GWG range with 7-11.5 kg for overweight and 5-9 kg for obese. ${ }^{5}$ The most important reason for this difference is that the IOM recommendation was derived largely from data collected among white women and may not well represent Chinese population. ${ }^{34}$ Therefore, we plan to establish GWG standards that can be applied to Chinese population.

Our data suggest effect modification by fetal sex in the association of GWG in late pregnancy with birth outcomes. Recent studies suggest that fetus sex may affect pregnancy outcomes. ${ }^{35} 36$ Although not very clear, how fetal sex may influence these outcomes may be explained by several factors. The placenta is an active endocrine organ, a sex-specific maternal-placental-fetal interaction may be involved. ${ }^{24}$ Animal studies suggest that maternal baseline BMI and GWG are associated with the hormonal milieu, including insulin resistance ${ }^{37}$ In agreement with this concept, a growing body of evidence link early pregnancy GWG with cord blood hormones that may affect fetal growth and development. ${ }^{38}$ Previous studies reported fetal sex differences in maternal first trimester hormones concentrations. ${ }^{153539}$ The resultant intrauterine environment may affect fetal development.

Our findings may have clinical implications. First, from early pregnancy onwards, GWG may affect subsequent maternal and neonatal outcomes. Second, although interventions to limit GWG in late pregnancy are effective, the benefits might be modest at best. To mitigate the harms of excessive weight gain, addressing the importance of gaining the appropriate amount of weight in both early and late pregnancy should be integrated into routine prenatal care. ${ }^{1740}$

\section{CONCLUSION}

The GWG associations with adverse pregnancy outcomes differ at early and late pregnancy, and there may be effect modification by fetal sex in the association of GWG in late pregnancy with some pregnancy outcomes. Weight gain management should be integrated into the routine prenatal care to decrease the risks of adverse pregnancy outcomes.

Acknowledgements We thank the study participants for permitting us to use their personal data.

Contributors YW and SW participated in interpretation of data and involved in drafting the manuscript. SG, ZM and LD analysed the data and critically revised the manuscript. ZL, JZ and XH made substantial contributions to conception and design, interpreted the data and critically revised the manuscript. All authors read and approved the final manuscript.
Funding This research was supported by a research grant (81873816) from the National Natural Science Foundation of China.

Competing interests None declared.

Patient consent for publication Not required.

Ethics approval This study was approved by Xinhua Hospital Research Ethics Committee, Shanghai Jiao Tong University School of Medicine (reference number: XHEC-F-NSFC-2018-122).

Provenance and peer review Not commissioned; externally peer reviewed.

Data availability statement Data are available upon reasonable request. Prospective scientists who are interested in the Shanghai Birth Cohort are welcomed to contact the authors via e-mail to (junjimzhang@sina.com).

Open access This is an open access article distributed in accordance with the Creative Commons Attribution Non Commercial (CC BY-NC 4.0) license, which permits others to distribute, remix, adapt, build upon this work non-commercially, and license their derivative works on different terms, provided the original work is properly cited, appropriate credit is given, any changes made indicated, and the use is non-commercial. See: http://creativecommons.org/licenses/by-nc/4.0/.

\section{ORCID iDs}

Zhongcheng Luo http://orcid.org/0000-0002-1794-1312

Xiaolin Hua http://orcid.org/0000-0003-1098-5010

\section{REFERENCES}

1 Goldstein RF, Abell SK, Ranasinha S, et al. Association of gestational weight gain with maternal and infant outcomes: a systematic review and meta-analysis. JAMA 2017;317:2207.

2 Cheikh Ismail L, Bishop DC, Pang R, et al. Gestational weight gain standards based on women enrolled in the fetal growth longitudinal study of the INTERGROWTH-21st project: a prospective longitudinal cohort study. BMJ 2016;352:i555.

3 Shulman R, Kottke M. Impact of maternal knowledge of recommended weight gain in pregnancy on gestational weight gain. Am J Obstet Gynecol 2016;214:.e751-754.

4 Krukowski RA, West DS, DiCarlo M, et al. Association of gestational weight gain expectations and advice on actual weight gain. Obstet Gynecol 2017;129:76-82.

5 Rasmussen KM, Yaktine AL, Institute of Medicine (US), National Research Council (US) Committee to Reexamine IOM Pregnancy Weight Guidelines. Weight gain during pregnancy: reexamining the guidelines. Washington (DC: National Academies Press., 2009.

6 Flegal KM, Kruszon-Moran D, Carroll MD, et al. Trends in obesity among adults in the United States, 2005 to 2014. JAMA 2016;315:2284-91.

7 Caughey AB. Gestational weight gain and outcomes for mothers and infants. JAMA 2017;317:2175-6.

8 Gaillard R, Steegers EAP, Franco OH, et al. Maternal weight gain in different periods of pregnancy and childhood cardio-metabolic outcomes. the generation R study. Int J Obes 2015;39:677-85.

9 Retnakaran R, Wen SW, Tan $\mathrm{H}$, et al. Association of timing of weight gain in pregnancy with infant birth weight. JAMA Pediatr 2018;172:136-42.

10 Sommer C, Mørkrid K, Jenum AK, et al. Weight gain, total fat gain and regional fat gain during pregnancy and the association with gestational diabetes: a population-based cohort study. Int $J$ Obes 2014;38:76-81.

11 Baci Y, Üstüner I, Keskin HL, et al. Effect of maternal obesity and weight gain on gestational diabetes mellitus. Gynecol Endocrinol 2013;29:133-6.

12 Cho E-H, Hur J, Lee K-J. Early gestational weight gain rate and adverse pregnancy outcomes in Korean women. PLoS One 2015;10:e0140376.

13 Zhang J, Tian Y, Wang W, et al. Cohort profile: the Shanghai birth cohort. Int J Epidemiol 2019;48:21-21g.

14 Johansson K, Hutcheon JA, Bodnar LM, et al. Pregnancy weight gain by gestational age and stillbirth: a population-based cohort study. BJOG 2018;125:973-81.

15 Collares FM, Korevaar TIM, Hofman A, et al. Maternal thyroid function, prepregnancy obesity and gestational weight gain-The generation R study: a prospective cohort study. Clin Endocrinol 2017;87:799-806.

16 Organization WH. Obesity: preventing and managing the global epidemic. Report of a WHO consultation, 2000. 
17 Berggren EK, Groh-Wargo S, Presley L, et al. Maternal fat, but not lean, mass is increased among overweight/obese women with excess gestational weight gain. Am J Obstet Gynecol 2016;214:e741-5.

18 Zhu L, Zhang R, Zhang S, et al. [Chinese neonatal birth weight curve for different gestational age]. Zhonghua Er Ke Za Zhi 2015;53:97-103.

19 Yee L, Caughey A, Cheng Y. Association between gestational weight gain and perinatal outcomes in women with chronic hypertension. Am J Obstet Gynecol 2017;09.

20 Waters TP, Dyer AR, Scholtens DM, et al. Maternal and neonatal morbidity for women who would be added to the diagnosis of GDM using IADPSG criteria: a secondary analysis of the hyperglycemia and adverse pregnancy outcome study. Diabetes Care 2016;39:2204-10.

21 Hutcheon JA, Bodnar LM, Joseph KS, et al. The bias in current measures of gestational weight gain. Paediatr Perinat Epidemiol 2012;26:109-16.

22 Laitinen J, Jääskeläinen A, Hartikainen A-L, et al. Maternal weight gain during the first half of pregnancy and offspring obesity at 16 years: a prospective cohort study. BJOG 2012;119:716-23.

23 Margerison-Zilko CE, Shrimali BP, Eskenazi B, et al. Trimester of maternal gestational weight gain and offspring body weight at birth and age five. Matern Child Health J 2012;16:1215-23.

24 Fraser A, Tilling K, Macdonald-Wallis C, et al. Association of maternal weight gain in pregnancy with offspring obesity and metabolic and vascular traits in childhood. Circulation 2010;121:2557-64.

25 Lawlor DA, Lichtenstein P, Fraser A, et al. Does maternal weight gain in pregnancy have long-term effects on offspring adiposity? A sibling study in a prospective cohort of 146,894 men from 136,050 families. Am J Clin Nutr 2011;94:142-8.

26 Starling AP, Brinton JT, Glueck DH, et al. Associations of maternal $\mathrm{BMI}$ and gestational weight gain with neonatal adiposity in the healthy start study. Am J Clin Nutr 2015;101:302-9.

27 MacDonald SC, Bodnar LM, Himes KP, et al. Patterns of gestational weight gain in early pregnancy and risk of gestational diabetes mellitus. Epidemiology 2017;28:419-27.

28 Tomedi LE, Simhan HN, Chang C-CH, et al. Gestational weight gain, early pregnancy maternal adiposity distribution, and maternal hyperglycemia. Matern Child Health J 2014;18:1265-70.

29 Dai ZY, Liu D, Li R, et al. [Association between gestational weight gain per trimester/total gestational weight gain and gestational diabetes mellitus]. Zhonghua Liu Xing Bing Xue Za Zhi 2016;37:1336-40.

30 Moore Simas TA, Waring ME, Callaghan $\mathrm{K}$, et al. Weight gain in early pregnancy and risk of gestational diabetes mellitus among Latinas. Diabetes Metab 2019;45:26-31.

31 Kominiarek MA, Saade G, Mele L, et al. Association between gestational weight gain and perinatal outcomes. Obstet Gynecol 2018;132:875-81.

32 Yee LM, Caughey AB, Cheng YW. Association between gestational weight gain and perinatal outcomes in women with chronic hypertension. Am J Obstet Gynecol 2017;217:e349:e341-8.

33 American College of Obstetricians and Gynecologists, Task Force on Hypertension in Pregnancy. Hypertension in pregnancy. Report of the American College of Obstetricians and Gynecologists' Task Force on Hypertension in Pregnancy. Obstet Gynecol 2013;122:1122-31.

34 Jiang $\mathrm{X}$, Liu M, Song $\mathrm{Y}$, et al. The Institute of medicine recommendation for gestational weight gain is probably not optimal among non-American pregnant women: a retrospective study from China. J Matern Fetal Neonatal Med 2019;32:1353-8.

35 Broere-Brown ZA, Schalekamp-Timmermans S, Hofman A, et al. Fetal sex dependency of maternal vascular adaptation to pregnancy: a prospective population-based cohort study. BJOG: Int J Obstet Gy 2016;123:1087-95.

36 Torricelli $\mathrm{M}$, Voltolini $\mathrm{C}$, Conti $\mathrm{N}$, et al. Weight gain regardless of pre-pregnancy $\mathrm{BMl}$ and influence of fetal gender in response to labor induction in postdate pregnancy. $J$ Matern Fetal Neonatal Med 2013;26:1016-9.

37 Nicholas LM, Rattanatray L, MacLaughlin SM, et al. Differential effects of maternal obesity and weight loss in the periconceptional period on the epigenetic regulation of hepatic insulin-signaling pathways in the offspring. Faseb $J$ 2013;27:3786-96.

38 Rifas-Shiman SL, Fleisch A, Hivert M-F, et al. First and second trimester gestational weight gains are most strongly associated with cord blood levels of hormones at delivery important for glycemic control and somatic growth. Metabolism 2017;69:112-9.

39 Arentson-Lantz EJ, Buhman KK, Ajuwon K, et al. Excess pregnancy weight gain leads to early indications of metabolic syndrome in a swine model of fetal programming. Nutr Res 2014;34:241-9.

40 Teede H, Harrison C, Lombard C. Case for action: proposal obesity prevention through preventing excess weight gain during pregnancy and postpartum, 2015 